\title{
Paläographische Übungen am Neujahrstag
}

\author{
von Stefan M. Maul - Heidelberg
}

Gelehrte und Schreiber, die in neuassyrischer Zeit in Assur Abschriften literarischer Keilschrifttexte anfertigten, versahen ihre Tafeln fast nie mit einem Datum. Die als KAR Nr. 111 veröffentlichte Tafel VAT $10382^{1}$ zählt zu den wenigen bekannt gewordenen Ausnahmen. ${ }^{2}$

Nabû-ețiranni, ${ }^{3}$ der noch in der Ausbildung stehende Sprößling einer Schreiber-Familie aus Assur, ${ }^{4}$ hatte diese Tafel mit einer säuberlichen Kopie des sog. Examenstextes $\mathrm{A}^{5}$ versehen und auf den Neujahrstag, den 1. Nisannu des Jahres 701 v. Chr. datiert. Seine „unter Druck“ (dulluhiš) angefertigte Abschrift versah er mit einem Kolophon, der so ungewöhnlich ist, daß er bislang in weiten Teilen nicht entziffert werden konnte. ${ }^{6}$

In der eigentlich konventionellen Tafelunterschrift finden sich nämlich nicht nur einige ungewöhnliche Graphien und selten gebrauchte Wörter. Der Kolophon ist auch in einem Schriftduktus verfaßt, der erheblich von dem Schrifttyp abweicht, in dem der ,Examenstext $A^{\star}$ niedergeschrieben worden war. Während der ,Examenstext $A^{6}$ - ohne jede Auffälligkeit in der neuassyrischen Schrift festgehalten wurde, die an der Wende vom

1 Die Fundumstände und die genaue Herkunft der Tafel sind unbekannt, da die auf der Ausgrabung vergebene Fundnummer verloren ging.

${ }^{2} \mathrm{Zu}$ diesen Ausnahmen gehören KAR 252 (VAT 9026; siehe H. Hunger, Babylonische und assyrische Kolophone [Kevelaer/Neukirchen-Vluyn 1968] 80 Nr. 236), geschrieben von dem jungen Schreiberlehrling (šamallû șehru) Nabû-šallim im Jahr 713 v. Chr.; KAR 267 (VAT 8237; siehe Hunger, Kolophone, 69 Nr. 199 B), geschrieben von Kișir-Aššur im Jahr 658 v. Chr.; LKA 37 (A 141; siehe Hunger, Kolophone 85 Nr. 259), geschrieben von Aššur-šuma-iškun im Jahr 734 (oder: 744?) v. Chr. und ferner die wohl in Ninive im Jahr 716 von Ninurta-uballissu, einem Bruder des Nabû-zuqup-kēnu, angefertigte Tafel KAR 174 (VAT 8807 = W. G. Lambert, Babylonian wisdom literature [Oxford 1960] Pl. 55-57; siehe Hunger, Kolophone, 96 Nr. 313). Außerdem ist die Notiz LKA 36 mit einem Datum versehen (A 162; siehe Hunger, Kolophone, 89 Nr. 290; datiert in das Jahr 733 v. Chr.).

3 Es ist eher unwahrscheinlich, daß dieser Nabû-ețiranni identisch ist mit dem Schreiber gleichen Namens, der eine Abschrift der Ritualtafel des Heilungsrituals Šurpu anfertigte (LKA 91 = VAT 13613).

4 Zu dieser Familie siehe den Kommentar zu Rs. 6'.

5 Siehe Å. W. Sjöberg, Der Examenstext A, ZA 64 (1975) 137-176 (dort Textvertreter J).

6 Siehe die Bearbeitung: Hunger, Kolophone, 78-79 Nr. 233. 
8. zum 7. Jh. üblich war, sind namentlich die ersten Zeilen des Kolophons überwiegend mit stark archaisierenden Zeichen geschrieben. Die von E. Ebeling als KAR 111 veröffentlichte recht genaue Zeichnung des Textes ließ dies in der gebotenen Deutlichkeit kaum erkennen, und so wird hier eine neue Autographie der Tafelrückseite vorgelegt.

Neben Zeichenformen, die sich eng an die sog., altbabylonische Monumentalschrift" anlehnen, ${ }^{7}$ gemahnen weitere altertümliche Keilschriftzeichen eher an mittelassyrische ${ }^{8}$ oder mittelbabylonische ${ }^{9}$ Konventionen. Darüber hinaus weist das Zeichen MU in Rs. 4' eine archaisch wirkende, aber wohl konstruierte Komplexität auf, wie sie nicht einmal in der Steleninschrift des ,Kodex Hammurapi` zu finden ist. ${ }^{10}$ In dem Kolophon findet sich darüber hinaus auch jene sehr auffällige Form des Zeichens A, ${ }^{11}$ die erst in neuassyrischer Zeit aufkam und vor allem aus den Steininschriften der Sargonidenkönige bekannt ist. ${ }^{12}$

Obgleich das Schriftbild des Kolophons auf den ersten Blick altertümlich erscheint, war die Vertrautheit des jungen Schreibers mit den alten Zeichenformen wohl noch nicht allzu groß. Denn in dem Kolophon finden sich nicht wenige Keilschriftzeichen, die stets unverändert im Stil der neuassyrischen Zeit belassen sind, ${ }^{13}$ obgleich auch für sie archaische Zeichenformen bekannt waren. Darüber hinaus fiel der junge Nabû-ețiranni immer wieder in die ihm weit besser von der Hand gehende neuassyrische Schrift zurück. So finden sich bisweilen neben den archaisierenden For-

7 AG (Rs. 5', 7'); AN (Rs. 3', 4' und passim); DUB (Rs. 7'); GAR (Rs. 3'); I (Rs. 3'); íl (Rs. 4'); LU (Rs. 3'); Ù (Rs. 3'); RA (Rs. 3').

${ }^{8}$ BA (Rs. $\left.3^{\prime}, 4^{\prime}, 5^{\prime}\right)$; вU (Rs. $\left.4^{\prime}\right)$; URU (Rs. $4^{\prime}, 5^{\prime}, 6^{\prime}, 9^{\prime}$ ).

9 E (Rs. 3').

${ }^{10}$ Eine nahezu identische Zeichenform ist freilich in einem spätbabylonischen Kultkalender belegt. Siehe S. M. Maul, Gottesdienst im Sonnenheiligtum zu Sippar, in: B. Böck u.a. (Hg.), Munuscula Mesopotamica. Festschrift für Johannes Renger. AOAT 267 (Münster 1999) 285-316, besonders $311 \mathrm{f}$.

11 In Rs. 3', 4', 5', 6' sowie in der Ligatur I+A (= IA; Rs. 6'). Zu dieser Form des Zeichens A siehe Maul, Tontafelabschriften des Kodex Hammurapi in altbabylonischer Monumentalschrift, ZA 102 (2012) 96 mit Anm. 53. Die eigentümliche Zeichenform des A findet sich auch in der Tafel VAT 13642 (+) VAT 13654 (D. Schwemer, KAL 2, Texte Nr. 4 (+) 5).

12 Der epigraphische Befund ähnelt damit sehr dem der von mir in ZA 102 vorgestellten Abschriften des Kodex Hammurapi.

${ }^{13} \mathrm{AZ}$ (Rs. 4', 5', 6'); DA (Rs. 6'); Di (Rs. 5'); É (Rs. 6', 7'); LI (Rs. 4', 7'); LÚ (Rs. 4', 5', 6') und Lư* (Rs. 3'ff.); MÁŠ (Rs. $4^{\prime}$ ff.); PEŠ (Rs. 6'); SAR (Rs. 3', 7'); SI (Rs. 5', 9'); SUM (Rs. 5'); ŠA (Rs. 5', $7^{\prime}$ ); ŠE (Rs. 6'); šÚ (Rs. $7^{\prime}, 8^{\prime}$ ); ŠUM (Rs. 3'); TA (Rs. 4' ff.); Ú (Rs. 3', 4' und passim); zI (Rs. 6'); zí (Rs. 6'). 
men von Keilschriftzeichen die jeweils zeitgenössischen. ${ }^{14}$ Die Lust, den Kolophon in einer altertümlichen Schrift zu notieren, hat den jungen Schreiber schon bald verlassen. Denn von Rs. $7^{\prime}$ an ist seine Handschrift von wenigen Ausnahmen abgesehen ${ }^{15}$ - wieder ganz im Stil seiner eigenen Zeit gehalten.

\section{VAT 10382, Rs.}

1'a [nam-dub-sar ĝiš-šub-ba sig ${ }_{5}$-ga] ' ${ }^{\prime}$ tuku' dr $1 a m m a^{\top}$ ${ }^{r}$ igi ${ }^{\top}{ }^{r}$ zálag $^{\top}-g[a]$

2'a [níg -šà-hab] ' 'é ${ }^{\top}-[\mathrm{g} a] 1-1 \mathrm{a}-\mathrm{ke}_{4}$

$1^{\prime} \mathrm{b} \quad$ [tup-šar-ru-tu i-siq da-ma-qi ra-še-e la-mas-si]

2'b [i-gu na-mi $] r$-[tu hišihti ekalli $]$

(Doppelstrich)

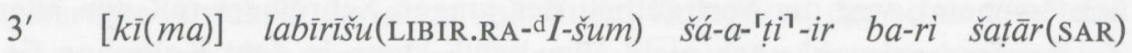

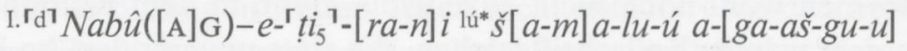

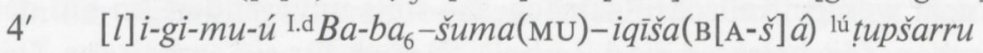

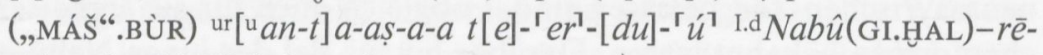

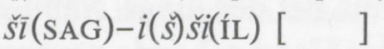

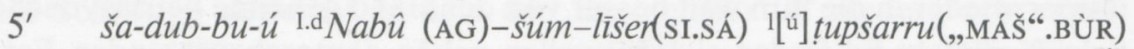

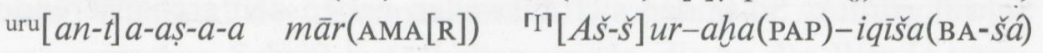
lúțup-šarru(xx) aššurû(BA[L.TIL $\left.\left.{ }^{\mathrm{ki}}-u\right]\right)$

6' $\quad m \bar{a} r(\mathrm{PEŠ})$ ă̌ Aš-šur-napišta(ZI)-še-și lút tupšarru(„MÁs“.BÙR) uru $a n-t a-$

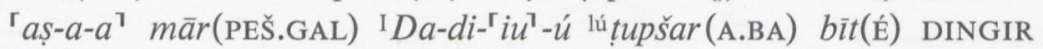
$[(\ldots)]$

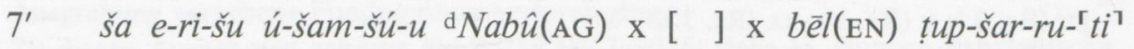
$\operatorname{sap} \bar{a} h$ (BIR)- $\breve{u}$ sapāh(BIR) bìtì(É)-šu li-i[ $[q-b i]$

$8^{\prime}$ ina qāt( $\mathrm{S̆} \mathrm{UI}^{\mathrm{II}}{ }^{\mathrm{d}}$ Gu-la a-zu-gal-la-tum rabītum(G[AL]-tum) murșu(GIG) la ' $p a^{\top}$-du-ú ina lib-bi-šú li[ $b$-ši $]$

9' itinisannu(BÁRA) UD.I.KAM lim-mu ${ }^{\mathrm{I}} \mathrm{Ha} a-n a-[n] a$ "uru“ $b \bar{e} l(\mathrm{EN})$ pāhat (NAM) uru „Til"“(DUL)-Bar-si-ib-[bi?]

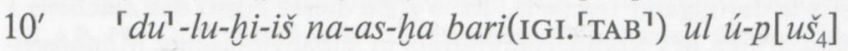

14 Vgl. das ,alte ${ }^{6} \mathrm{~A}$ in Rs. 3', 4', 5', 6' mit der neuassyrischen Form in Rs. 6' (in: lù A.BA) und in Rs. $8^{\prime}$ (in: a-zu-gal-la-tum); vgl. das ,alte' AN in Rs. $3^{\prime}, 4^{\prime}, 5^{\prime}$ mit der neuassyrischen Form in Rs. 6', 7', 8'; vgl. das ,alte' BA in Rs. 3', 4', 5' mit der moderneren Form in Rs. 6' (in: lùA.BA); vgl. das ,alte' $\mathrm{E}$ in Rs. $3^{\prime}$ mit der neuassyrischen Form in Rs. $7^{\prime}$; vgl. das ,alte DUB in Rs. 7 mit der neuassyrischen Form in Rs. 5'; vgl. das ,alte' LU in Rs. $3^{\prime}$ mit der neuassyrischen Form in Rs. 10'.

15 AG (Rs. 7'); DUB (Rs. 7'); URU (Rs. 9'). 


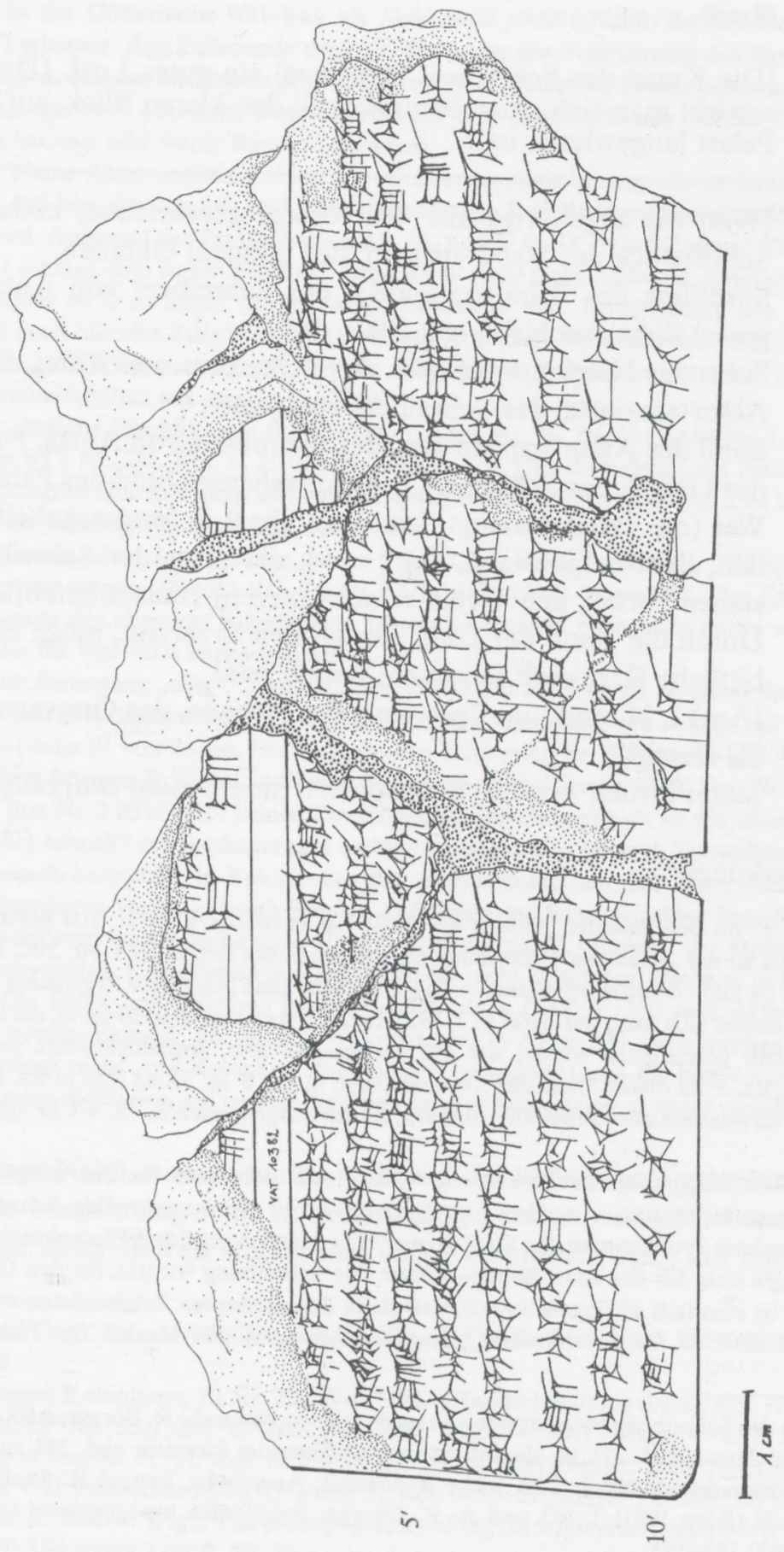


(Rand)

Rs.1'f. [Die Kunst des Schreibers (beschert) ein gutes Los]. (Durch sie) erwirbt man sich einen Schutzengel: den klaren Blick, auf den der Palast [angewiesen ist]!

3' [Wie] die zugehörige alte Textvorlage geschrieben, kollationiert. Schriftstück des Nabû-ețiranni, des [jungen] Gehilfen,

4' Sprößling des Bāba-šuma-iqiı̌sa, des Schreibers von Antaș, (jüngerer) Sohn des Nabû-rēšī-iš(̌̌)i, des [ ],

5' Sohn des Nabû-šum(u)-līšer, des Schreibers von Antaș, Sohn des Aššur-aha-iqī̌sa, des Schreibers von Assur,

6' Sohn des Aššur-napišta-šēși, des Schreibers von Antaș, Nachfahre des Dādijû, des Schreibers des Gotteshauses [und des Palastes (?)].

7' Wer (die Tafel) verlangt, (sie dann aber) in Vergessenheit geraten läßt, dem möge Nabû, der [ ], der Herr der Schreiberkunst, seinen Verfall, den Verfall seines (ganzen) Hauses befeh[len].

8' Durch die Hand der Gula, der großen Erzärztin, möge eine unerbittliche Krankheit in seinem Bauch sein!

9' (Am) 1. Nisannu im Eponymat des Hanana, des Gouverneurs von Til-Barsip, ,unter Druck‘ kopiert, kollationiert, (doch) nicht end[gültig geprüft].

\section{Anmerkungen}

Rs. 3' Im beschädigten Anfang der Zeile hat entweder $k i$ oder GIM gestanden. Eine Parallele zu der ,gelehrten' Schreibung für labirǐ̌su findet sich in STT Nr. 300, Rs. 21: GIM ${ }^{\mathrm{d}}$ LAMA $(=l a b)$-d $\dot{E} r-r a(=\bar{i} r \grave{i})$-d $I$-šum $(=\bar{i} \breve{s} u)$. Die nicht ganz gesicherte Ergänzung des Zeilenendes richtet sich nach der Tafel STT 192, Rs. 24' (lúšAB.TUR a-ga-aš-gu-u), die in dem gleichen Jahr geschrieben wurde, wie der vorliegende Text. Auffälligerweise weist in VAT 10382, Rs. $3^{\prime}$ an dieser Stelle das A (so wie auch in Rs. 6' in: lùA.BA und in Rs. 8') nicht die sonst verwendete altertümelnde, sondern die im ausgehenden 8 . Jh. v. Chr. übliche Form auf.

4' Die logographische Schreibung lú,MÁs“.BÙR steht hier für das selten gebrauchte Logogramm lúGI.BÙR = tupšarru. ${ }^{16}$ uru $A n$-ta-aș(-șa) ist ein im normalen Schrifttum kaum verwendeter Prunknamen der Stadt Assur. ${ }^{17}$ Die hiervon gebildete Einwohnerbezeichnung antașajju steht für das sonst übliche aššrû. Die Schreibung ${ }^{\mathrm{d}} \mathrm{GI}$.HAL für den Götternamen Nabû ist ebenfalls so ungewöhnlich, daß sie in den modernen Zeichenlisten nicht gebucht ist. In einer in Gelehrtenkreisen bekannten Liste mit den Namen des Nabû (V R 43

$16 \mathrm{Zu}$ der Schreibung lúMÁŠ.BÙR mit Verweis auf lúGI.BÙR siehe R. Borger, ABZ 78 zu Nr. 76 und ders. ${ }^{2}$ MZL 276 zu Nr. 130, mit weiterführender Literatur ebd. 281 zu Nr. 141.

17 ,Götteradressbuch‘ Z. 189: Siehe B. Menzel, Assyrische Tempel II. Studia Pohl s.m. 10/II (Rom 1981) T 165 und A. R. George, Babylonian topographical texts (Leuven 1992) $182-183$. 
Rs. 32$)^{18}$ ist der Göttername d Gi-hual als Nabû bānû pirišti („Nabû, der das Geheimnis erschafft") erläutert. Am Zeilenende muß ein Titel oder die Bezeichnung des Berufes gestanden haben, den der Großvater des Nabû-ețiranni ausgeübt hat (wohl: țupšarru; für den Zusatz antașajju bzw. aššurû ist, wenn er nicht auf dem abgebrochenen rechten Tafelrand gestanden hat, nur sehr wenig Raum vorhanden).

6' Der Name Aššur-napišta-šēși ist hier offenbar erstmals bezeugt. Es ist ferner ungewöhnlich, daß hier der liegende und nicht der stehende Keil als Personendeterminativ gebraucht wird. Aufgrund der Parallelstelle KAR 25, Kol. iv 9'-11' ( ${ }^{1} D a-[d] i-i u$-ú / lú* DUB.SAR

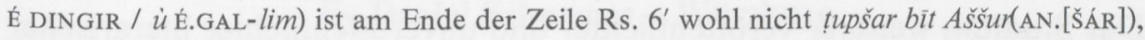
sondern tupšar bìt ili zu lesen. Die Raumverhältnisse in VAT 10382 nähren den Zweifel daran, daß auch hier der Zusatz $u$ ekalli $(m)$ folgte. Von allen Vorfahren des Nabû-ețiranni ist uns allein Dādijû bekannt. Er galt, wie wir aus weiteren Kolophonen wissen, ${ }^{19}$ auch anderen Schreiberfamilien aus Assur als Ahnherr.

7' Sehr ähnliche Fluchformeln finden sich in: LKA Nr. 76, Rs. 16; BAM Nr. 1, Kol. iv 34 sowie in STT Nr. 215, Kol. vi 21 und Nr. 247 Rs. 20. Das erste dem Gott Nabû zugewiesene Epitheton läßt sich nicht mehr rekonstruieren. Vielleicht ist $\mathrm{E}[\mathrm{N}(\mathrm{x})] \mathrm{x}$ zu lesen.

$8^{\prime}$ Vgl. die Parallelstelle KAR 252, Kol. iv 54.

9' Zu Hanana, dem Eponym des Jahres 701 v. Chr., siehe A. Millard, The eponyms of the Assyrian empire 910-612 BC. SAAS 2 (Helsinki 1994) 49 und 94. Das Ende der Zeile $9^{\prime}$ ist nach den ebendort aufgeführten Textstellen ergänzt. uruEN-NAM dürfte wohl als Schreibfehler für lúEN-NAM anzusehen sein.

$10^{\prime}$ In der Bedeutung „eilig“, „unter großem Druck“ ist dulluhiš nur an dieser Stelle belegt. Die in den akkadischen Synonymenlisten bezeugte Gleichung von dulluhu mit urruhu und hamātu (siehe W. von Soden, Die lexikalischen Tafelserien der Babylonier und Assyrer in den Berliner Museen II: Die akkadischen Synonymenlisten [Berlin 1933] Text Nr. 1 Kol. v $23 \mathrm{f}$. und Text Nr. $2231 \mathrm{f}$.) läßt keinen Zweifel daran, daß dulluhiš hier, so wie urruhiš und hantišs, „(sehr) schnell“ o.ä. bedeutet und nicht etwa wie in den übrigen Textstellen „ganz verstört“. uppušu bezeichnet in Kolophonen der spätbabylonischen Zeit einen noch nicht genau bestimmbaren Vorgang, durch den das Anfertigen und Kopieren einer Tontafel endgültig abgeschlossen wurde (siehe dazu Hunger, Kolophone, 3). Obgleich der Stativ uppuš in den neuassyrischen Kolophonen aus Assur bislang nicht belegt war, dürfte er in Rs. 10' vorliegen. Die Folge nasha/šatirma bari (u) uppuš (siehe CAD E 232 und AHw. 227) ist nämlich bestens bezeugt. So wie von W. von Soden in AHw. 227 angenommen, kann uppušu allerdings nicht „mit Kolophon abschließen“ bedeuten. Denn die hier vorgestellte Tafel weist einen Kolophon auf und ist dennoch mit der Bemerkung ul uppuš versehen.

Der junge Nabû-ețiranni wollte wohl mit der kleinen paläographischen Übung am Neujahrstag seine in seinen Augen schon weit gediehene Gelehrsamkeit unter Beweis stellen und dabei vermutlich auch auf das ver-

18 Siehe auch D. O. Edzard/W. G. Lambert, R1A III (Berlin/New York 1957-1971) 356 s.v. Gihal.

19 Siehe Hunger, Kolophone, 79 Nr. 235 (VAT 9823 [Handerhebungsgebete], Kol. iv 3-16 [Kopie: KAR Nr. 23]) und 83 Nr. 246 (VAT 10262 + VAT 12965 + VAT 12973 [Erim-huš = anantu Tf. 6], Kol. iv 25-28 [Kopie: A. Cavigneaux, FuB 20/21 (1980) 265-267]; VAT 9000 [Uru-an-na Tf. 2 und 3], Kol. iv 40-51 [KADP Nr. 11]). Zu Dādijû siehe auch: K. Radner (Hg.), The prosopography of the Neo-Assyrian empire 1/II (Helsinki 1999) 364 sowie TIM 9, Nr. 56. 
meintlich oder tatsächlich hohe Alter des von ihm studierten Textes hinweisen. ${ }^{20}$ Damit folgte er aber wohl kaum einer eigenen spontanen Idee, sondern vielmehr einer bislang keineswegs gut dokumentierten Schreibergepflogenheit der neuassyrischen Zeit. Denn mit VAT 14046 (KAL 4 Nr. 5) kennen wir eine andere von einem jungen Schreiber (šamallû șehru) angefertigte Tafel, deren Kolophon in einer von dem Haupttext erheblich abweichenden, sehr altertümlichen Schrift niedergeschrieben wurde. ${ }^{21}$ Das Studium weiterer veröffentlichter und unveröffentlichter literarischer Texte aus Assur und deren möglichst genaue epigraphische Dokumentation wird uns neue Beispiele für derartige paläographische Übungen junger Schreiber liefern. ${ }^{22}$

20 Als Hinweis auf das hohe Alter der Textvorlage läßt sich in literarischen Keilschrifttexten des 1. Jt. auch das Einstreuen alter Zeichenformen in einen in zeitgenössischer Schrift gehaltenen Fließtext immer wieder beobachten (vgl. z. B. D. Schwemer, Rituale und Beschwörungen gegen Schadenzauber. Keilschrifttexte aus Assur literarischen Inhalts 2 [Wiesbaden 2007] 9 zu Text Nr. 4 und Maul, Fs. J. Renger, 311f.).

21 Siehe dazu: S. M. Maul/R. Strauß, Ritualbeschreibungen und Gebete. Keilschrifttexte aus Assur literarischen Inhalts 4 (Wiesbaden 2011) 32 zu Text Nr. 5, Rs. 11'f.

22 Die vorliegende Studie zeigt sehr deutlich, daß die Datierung eines Textes möglichst nicht allein aufgrund der in einem Kolophon belegten Zeichenformen erfolgen und stets auf einer Untersuchung aller in dem Text vorkommenden Zeichenformen fußen sollte. 